ALPHA No 26 Julio / 2008 (261-279)

ISSN 0716-4254

http://alpha.ulagos.cl

\title{
PRODUCIR UNA EMPRESA EDITORIAL. EL CASO DE LA REVISTA DE CRÍTICA CULTURAL EN CHILE
}

Producing an editorial Enterprise. The Revista de Crítica Cultural case en

Chile

Carolina Ramírez Álvarez*

\section{GENEALOGÍA CULTURAL: "ESCENA DE AVANZADA"}

El primer número de la Revista de Crítica Cultural sale en Chile a la luz pública en mayo de 1990, fecha que sobresale de manera significativa por la carga simbólica que puede atribuírsele. Este dato temporal apunta a un momento de quiebre que evidencia, por lo menos de una forma aparente, una línea divisoria entre el pasado militar y el presente democrático de Chile. El 11 de marzo de 1990, Augusto Pinochet entrega — gracias a los resultados del plebiscito de $1988^{1}$ y las elecciones presidenciales de 1989- el mandato a Patricio Aylwin, primer jefe de Estado electo luego de diecisiete años de dictadura. Por estas circunstancias, es imposible pasar por alto la fecha de la fundación de la revista. Al contrario, la aparición del número inaugural dos meses más tarde del cambio de gobierno es un elemento que interpela al lector ¿por qué aparece en ese momento álgido de la historia chilena una "revista de crítica cultural”? Cuando sale ese primer número ¿se piensa que se vive un período ruptural? ¿Es sintomático -o no lo es- su inicio en una época como ésa? Por supuesto, son varias las respuestas que podrían derivarse de estas interrogantes. Sin embargo, la respuesta que interesa explorar para el desarrollo de este trabajo es la que parte de la premisa de que esta Revista de Crítica Cultural trata de ser identificada, en un primer momento, con un tiempo histórico nuevo: un tiempo presente que se critica, que revisa el pasado y que imagina un futuro posible ${ }^{2}$.

${ }^{1}$ El plebiscito fue llevado a cabo el 5 de octubre de 1988. Consistía en preguntar a la ciudadanía si estaba a favor o en contra de la continuidad del régimen militar. Los resultados fueron favorables para quienes deseaban elecciones presidenciales para el próximo año. El "sí", con $44 \%$ de los votos, sucumbió ante el "no" que obtuvo el 55,7\%. En consecuencia, fue posible convocar a elecciones en diciembre de 1989, donde Patricio Aylwin resultó elegido Presidente de la República de Chile.

${ }^{2}$ Aquí hago un juego de palabras con Revisar el pasado, criticar el presente, imaginar el futuro (2004), libro editado por Nelly Richard que debe su nombre al Coloquio Internacional realizado, en el Centro de Convenciones Diego Portales (4, 5 y 6 de septiembre de 2003) en 


\section{Carolina Ramírez Álvarez}

Pensar el proceso de producción de la Revista de Crítica Cultural (1990) significa, primero, echar una mirada sobre la fecha de su fundación porque, como se mencionó antes, surge justo en un cambio político fundamental para Chile: el "Tránsito formal de un régimen a otro", tal y como lo sostienen Marcela Ríos, Lorena Godoy y Elizabeth Guerrero (2003). Y esto implica "mucho más que el cambio de instituciones y procedimientos políticos" debido a que marca "el comienzo de una nueva etapa para la sociedad en su conjunto" porque se trata

de una profunda transformación de la vida política, de las formas de interacción entre el Estado y los actores sociales, del sistema de representación de intereses y de las dinámicas de acción colectiva. Se trata, también, de cambios en el orden simbólico, que afectan y son afectados por el modo en que se interpretan tales transformaciones, así como las nuevas formas de hacer política. De este modo, hablar de transición es hablar, también, de un tránsito en las formas de organización social, en la interacción de la sociedad con el sistema político, y sobre todo, en los imaginarios y los discursos políticos que sustentan el quehacer de los actores dentro y fuera de las esferas políticas formales (2003:23).

Sin lugar a dudas, el contexto de surgimiento de la Revista de Crítica Cultural, a primera vista, se presentaba como un tiempo que abría las posibilidades reales de discusión, que le permitía a los actores sociales participar de los nuevos imaginarios que el sujeto chileno debía construir. Asimismo, era un tiempo propicio para el debate cultural porque, como lo afirma Olea, la "transición es, sobre todo, un tiempo de incertezas, de interrogantes y también de apertura y búsqueda, a otros modos de hablar”. (S.D.) $)^{3}$

Santiago de Chile. El evento se organizó con la idea de reflexionar sobre el aniversario de los treinta años del golpe militar (11 de septiembre de 1973).

${ }^{3}$ Subrayo la expresión "a primera vista” para dejar sentado que, a partir de la propuesta del sociólogo Tomás Moulián, existe una puesta en duda de las interpretaciones de la "transición democrática” chilena. Según Moulián, el tránsito del régimen dictatorial a uno democrático no se inició cuando Pinochet entregó el mando presidencial sino que hubo un "largo proceso de preparación, durante la dictadura, de una salida de la dictadura, destinada a permitir la continuidad de sus estructuras básicas bajo otros ropajes políticos, las vestimentas democráticas. El objetivo es el "gatopardismo", cambiar para permanecer. Llamo "transformismo" a las operaciones que en el Chile actual se realizan para asegurar la reproducción de la "infraestructura" creada durante la dictadura, despojada de las molestas formas, de las brutales y de las desnudas "superestructuras” de entonces. El 'transformismo' consiste en una alucinante operación de perpetuación que se realizó a través del cambio del Estado. Éste se modificó en varios sentidos importantes, pero manteniendo inalterado un 


\section{Producir una empresa editorial. El caso de la Revista Crítica Cultural}

Luego de comprender la coyuntura histórica en que surge la revista, es necesario prestar atención a un momento anterior al de su aparición debido a que, como lo sostiene su directora, Nelly Richard (en una entrevista personal, 2005), "un antecedente clave en la formulación del proyecto editorial [...] se sustenta en los años ochenta con una "escena de avanzada” que se localiza, principalmente, en torno a las artes visuales". Esto quiere decir que para comprender el impulso que dio vida a la publicación es importante revisar una tendencia artística que tuvo lugar en tiempos dictatoriales; tiempo en el que, por supuesto, la Revista de Crítica Cultural no se había concretado como un producto material.

Nelly Richard, en la entrevista en referencia, declara que la "escena de avanzada” constituye el antecedente inmediato de la publicación, puesto que ésta "nace como una especie de prolongación y de rearticulación" de ese conjunto de manifestaciones culturales que tuvieron lugar en los años de dictadura y que fueron aglutinadas, posteriormente, bajo ese nombre. Es decir que con el proyecto de la revista se trata de continuar una labor de productividad creativa que, por las condiciones de surgimiento, se había manifestado de forma "intensa y profusa" y, al mismo tiempo, de forma "fragmentaria y dispersa". Por ello, con el proyecto editorial se intenta construir un escenario que sistematice y dé cuerpo a un discurso ruptural que emergió como respuesta a un régimen político (la dictadura militar de Pinochet) que limitaba las producciones artísticas en el campo cultural chileno post $73^{4}$. En palabras de Nelly Richard, el proyecto editorial tenía entre sus

aspecto sustancial. Cambia el régimen de poder, se pasa de una dictadura a una cierta forma de democracia y cambia el personal político en los puestos de comando del Estado” (141). En efecto, el régimen dictatorial fue armando un terreno de salida que le permitiera, a su vez, una actuación directa en la nueva democracia. Por ello, Moulián no habla de "transición" sino de "transformismo", es decir, se mantiene el régimen organizacional empleado en la dictadura pero, ahora, con "nuevos ropajes democráticos". En este punto es ilustrativo el hecho de que Pinochet, luego de su salida del cargo de Presidente de la República (1990), se mantuviera como Comandante en Jefe del Ejército hasta 1998, situación “que manifestaba su privilegio por sobre todos los demás” y que, además, confirmaba que sus acciones podrían ser realizadas, nuevamente, "a través del ejercicio de la fuerza y de la amenaza de golpe” (Uribe, 1998:40). Pinochet, sin poseer el mandato presidencial, quedaba como cabeza de las Fuerzas Armadas de Chile; el mismo cargo que le permitió derrocar al gobierno de Allende en 1973.

${ }^{4}$ Obviamente, las limitaciones impuestas por la dictadura no son sólo en el campo artístico. Por ejemplo, Faride Zerán expone el impacto del régimen militar en los medios de comunicación: "El 11 de septiembre de 1973, en medio del Estado de Sitio decretado en todo el país, desde tempranas horas eran bombardeadas las antenas transmisoras y se confiscaban los equipos de radios Magallanes, Corporación, Luis Emilio Recabarren y Nacional. En el bando $\mathrm{N}^{\mathrm{o}} 10$, se conminaba a entregarse voluntariamente en el Ministerio de Defensa a 94 personas, entre las cuales estaban los periodistas Eugenio Lira Massi, José Miguel Varas, Carlos Jorquera, Augusto Olivares, Frida Modak, Mario Gómez López, Fernando Rivas Sánchez, René Olivares 


\section{Carolina Ramírez Álvarez}

objetivos "darle fuerza a ese potencial crítico y, por otro, tratar de articular un soporte material" que estuviera "acorde con el cambio de paisaje, de posibilidades, de subjetividades" que la transición democrática prometía ${ }^{5}$. En suma, la publicación surgía — junto con la "transición democrática”- con el deseo de continuar una labor artística/crítica que se había producido con el objeto, en palabras de Diamela Eltit, de "explicitar apasionadamente el malestar, la crítica, la abierta disidencia no sólo con la realidad dictatorial sino, además, con otras prácticas artísticas” (2004:102).

Ahora bien, es relevante señalar que al realizar esta mirada al pasado cultural - por parte de los editores de la revista y, en especial, por la directora - se está operando con una doble intencionalidad: la de legitimar cierta forma de creación y, gracias a la inscripción en una práctica precedente, legitimar el lugar desde el cual se arma el discurso. Es decir, la revista -a través del relato de su directora- se encarga de elaborar una ficción de linaje que le permite construir una genealogía cultural que afilia su proyecto a un tiempo anterior; tiempo que da un asidero histórico a una naciente revista. En este sentido, es contundente el texto de presentación que aparece en la página web de la publicación (www.criticacultural.org), debido a que allí, de forma directa, se manifiestan las deudas de la empresa editorial con esa "escena" caracterizada, según Richard, por practicar una

y Juan Ibáñez”. En el bando № 11, de ese mismo día, la Junta de Gobierno señalaba su deseo de "mantener informada a la opinión pública sobre acontecimientos nacionales"; agregando inmediatamente después: "De acuerdo con lo dispuesto en los bandos hasta ahora emitidos y por encontrarse el país en Estado de Sitio, se ha dispuesto ejercer sobre los medios de comunicación publicados una estricta censura de prensa. Como una primera medida precautoria durante el día 12 de septiembre de 1973 se ha autorizado solamente la emisión de los siguientes diarios: El Mercurio y La tercera de la Hora". "Se ha designado una Oficina de Censura de Prensa que funcionará en la Academia Politécnica Militar del Ejército” — señalaba a continuación el bando- Mientras tanto, eran clausurados los diarios El Clarín, Noticias de Última Hora, El Siglo, Puro Chile; las revistas Mayoría, Paloma, Hechos Mundiales, Onda, Saber para todos, El Manque, Chile Hoy, Ramona y Punto Final y se cerraban las agencias informativas Prensa Latina, de Cuba, y CTK, de Checoslovaquia. El día 12 de septiembre otros periodistas eran llamados a 'entregarse voluntariamente' y en el bando $\mathrm{N}^{\circ} 24$ se exponía 'para conocimiento de la ciudadanía’ la ocupación de los diarios La Nación y Clarín, de la revista Punto Final y de la editorial Quimantú" (2004:210).

5 Para darse una idea de lo que significa el cambio político en los diversos medios de comunicación, Guillermo Sunkel narra que "La emergencia de experiencias radiales de base que transmitían esporádicamente desde diversos puntos poblacionales, especialmente en Santiago, es un fenómeno que coincide con el retorno a la democracia en Chile. En efecto, es en enero de 1990, a menos de un mes del triunfo de la Concertación, cuando medio centenar de “equipos de radialistas independientes", vinculados a programas de comunicación social de ONG's, organizaciones sociales y de Iglesia dan vida a la Agrupación Nacional de Radio Popular - ANARAP” (200). 


\section{Producir una empresa editorial. El caso de la Revista Crítica Cultural}

escritura teórica informal, desensamblada, que se sentía atraída por la vagancia de conceptos sin ataduras de género ni estrictas filiaciones disciplinarias, y que transitaba por las zonas de emergencia de prácticas artísticas y literarias igualmente riesgosas, fuera de las vigiladas fronteras del saber académico (2006).

Luego de la descripción de las formas de producción de la "escena", Richard no duda en afirmar que fue ese "antidisciplinamiento teórico de la escena de escrituras que guió el surgimiento de la Revista de Crítica Cultural”. Lo que interesa para esta argumentación es dejar sentadas las conexiones que traza Richard con un pasado cultural que ella misma articula. Esto quiere decir que la directora de la revista se incluye como una de las figuras capitales en el relato que erige porque, como se verá a continuación, ella es quien, prácticamente, lo organiza. Gran parte de su trabajo crítico está centrado en la "escena de avanzada" que es "una especie de visión de conjunto, de mirada totalizante, de todo un fenómeno que se" vivió "bajo la forma de eventos aislados y de lo disperso" (Valdés,1987: 81). Esto significa que Nelly Richard ordena un discurso que, según Valdés, Oyarzún y ella misma, se presentaba como diseminado. Por ello, Pablo Oyarzún advierte que en Márgenes e instituciones. Arte en Chile desde 1973 - libro fundador del trabajo de Nelly Richard, 1986) - "la crítica se convierte en historia” porque

rescata la producción de la "escena de avanzada” (y se rescata a sí misma como su testigo más fiel, y como algo más que un simple testigo...), que viene a historizar indefectiblemente a esta "escena", a sancionarla, como pretérito, a convertirla en testimonio de sí misma, con el efecto monumental que de ello, quiéraselo o no, se sigue (1987:45. Énfasis del autor).

Según Oyarzún, la directora trata de recrear un fragmento de la historia cultural chilena y, por ende, funda uno nuevo ${ }^{6}$. Bernardo Subercaseaux, en esta línea de pensamiento, sostiene que

\footnotetext{
${ }^{6}$ Es importante señalar que al construir un discurso relativamente ordenado de un movimiento cultural — cualquiera que sea — se hace, a su vez, una historia que puede resultar parecida a los grandes relatos que se critican. En este sentido, Benjamin sostiene que "al pasado sólo puede retenérsele en cuanto imagen que relampaguea” (1973:180), es decir, como un dibujo que nunca puede ser aprehendido por su persistente movilidad. Rememorar es un ejercicio llevado a cabo gracias a las latencias del pasado; son los relampagueos de esa imagen, las figuras que se repiten las que hablan, o mejor dicho, a las que se les hace hablar. Esas ruinas, en tanto despojos de una época, iluminan una pequeña porción de lo ocurrido. Es de esta forma, entendiendo que esa "recuperación” es también una re/elaboración de un fragmento de la historia, que debiera leerse la producción crítica de Richard.
} 


\section{Carolina Ramírez Álvarez}

en el caso de Márgenes e instituciones... muchos de sus rasgos más peculiares obedecen a las necesidades internas de productividad del propio discurso. Digamos, en primer lugar, que se trata de un discurso de tendencia y que, por lo tanto, se propone no sólo hacer inteligible a un sector de la producción artística (la neovanguardia o avanzada) sino que también articular y poner en escena su supremacía dentro de la década (1987:36).

Visto así, queda de manifiesto que Richard construye un relato en el que ella y su objeto de estudio resultan protagonistas; personajes principales de una época en la que existieron múltiples producciones culturales que fueron opacadas por el "propio discurso" de la Directora de la publicación. Según Subercaseaux, con el discurso de Richard no sólo se pretende privilegiar el lugar de la "avanzada" sino el lugar de producción del discurso que toma como objeto de estudio a esa "escena". Por esta razón, el quehacer editorial que supone la revista seguirá con una de las vertientes teóricas de su fundadora: privilegiar los discursos que tengan conexión con las producciones que el relato de Richard favorece.

En el horizonte discursivo de la Revista de Crítica Cultural, la "escena de avanzada” agrupa a las creaciones que emergieron "en plena zona de catástrofe” cuando naufragó

el sentido, debido no sólo al fracaso de un proyecto histórico, sino al quiebre de todo el sistema de referencias sociales y culturales que, hasta 1973, articulaba — para el sujeto chileno- el manejo de sus claves de realidad y pensamiento (Richard, 1987:2).

Esa escena de explosión cultural que surge a partir de 1977 — desde la plástica (Eugenio Dittborn, Carlos Leppe, Juan Dávila, Carlos Altamirano, el grupo $\mathrm{CADA}^{7}$, Lotty Rosenfeld, Catalina Parra, Alfredo Jaar, etc.) y desde la literatura (Raúl Zurita, Diamela Eltit, Diego Maquieira, Juan Luis Martínez, Gonzalo Muñoz, Soledad Fariña, etc.) - se apartaba de las estéticas dominantes que promovían trabajos de fuerte raigambre política y que tendían a clasificar la realidad en dicotomías. Desde luego, el distanciamiento hacia

\footnotetext{
${ }^{7}$ El Colectivo de Acciones de Arte (CADA) realizó diferentes perfomances en Santiago, durante la dictadura. Las "acciones" criticaban, si no de manera explícita, de forma tangencial el terrorismo institucionalizado. CADA estaba compuesto por destacados personajes del quehacer cultural chileno, entre ellos, Diamela Eltit, Juan Castillo, Fernando Balcells, Raúl Zurita y Lotty Rosenfeld. Cfr. Robert Neustadt (2001).

${ }^{8}$ Me guío aquí por la clasificación utilizada por Nelly Richard en el capítulo "Una cita limítrofe entre neovanguardia y postvanguardia”, específicamente en la nota número 3 , del libro $L a$ insubordinación de los signos (1994).
} 


\section{Producir una empresa editorial. El caso de la Revista Crítica Cultural}

los discursos partidarios de contenidos explícitos no quiere decir que las producciones culturales de "la avanzada" estuvieran al margen de posicionamientos políticos determinados pero, al menos, se encontraba latente la intención de librarse de las clasificaciones reductoras. Para Richard, esta escena representó un quiebre profundo en la forma de hacer arte y, por ello, "el proyecto de la revista tenía que ver con la idea de potenciar la energía crítica que se generaba” a su alrededor (entrevista personal, 2005). En consecuencia, la publicación se fundaba con la idea de retomar un trabajo que se había iniciado en los años de la dictadura pero que, por diversas razones, no había tenido las posibilidades de recogerse sistemáticamente. El discurso crítico que se pretendía elaborar desde las páginas de la revista, como desde el trabajo efectuado por quienes intentaban repensar el pasado contribuía con la recuperación, por tanto, con la legitimación de esas creaciones y con la valoración del discurso teórico que se edificó en torno a ellas.

\section{FUNDADORES QUE IMPULSAN EL PROYECTO EDITORIAL}

Como se argumentó anteriormente, la Revista de Crítica Cultural tiene sus antecedentes en la "escena de avanzada” ya que, cuando se concretó la idea de la publicación, participaron algunos de sus protagonistas (Richard, entrevista personal, 2005). Nelly Richard, Juan Dávila (artista y escritor), Eugenio Dittborn (artista visual), Diamela Eltit (escritora y ex miembro del grupo CADA), Carlos Pérez (filósofo), Adriana Valdés (ensayista) y Carlos Altamirano (artista plástico) fueron los encargados de dar vida al proyecto editorial en sus inicios. Ellos, según la directora, protagonizaron la "escena” y son quienes figuran, hasta el número diez, como parte del consejo editor ${ }^{9}$.

Casi todos los nombrados colaboran en forma directa en el primer número. Es decir, no sólo resaltan por ser los “editores” sino, también, por ser los "escritores/artistas", quienes ayudan a construir la entrega inaugural y, con ello, a fundar el proyecto. Todos tienen un espacio en ese número y la mayoría lo ocupa para avalar los trabajos de sus compañeros o, bien, para legitimar producciones que dialogan con la línea editorial de la revista. Por ejemplo, se publica una ponencia de Richard que fue presentada en un seminario internacional, llevado a cabo en Valparaíso (Chile) un mes antes de la entrada en circulación de la revista ${ }^{10}$. Este artículo, titulado "Estéticas de la

\footnotetext{
${ }^{9}$ El consejo editorial que fundó la Revista de Crítica Cultural se mantuvo intacto hasta el № 10 (mayo de 1995). Luego de ese número comienzan las variaciones; por ejemplo, en el $\mathrm{N}^{\circ} 11$ (noviembre 2005) sale la figura de Dittborn y, en el $\mathrm{N}^{0} 12$ (julio de 1996), sale Carlos Altamirano y se incluye a Willy Thayer.

${ }^{10}$ Dato que vale la pena acentuar porque es una constante que en la Revista de Crítica Cultural se recopilen materiales que han sido leídos o publicados en otros ámbitos. Característica que se
} 


\section{Carolina Ramírez Álvarez}

oblicuidad”, es capital debido a tres razones: la primera de ellas porque corresponde al escrito de la directora y su inclusión puede leerse como un gesto de autoridad que anuncia su actuación en la conformación del proyecto; la segunda razón tiene que ver con el funcionamiento del escrito dentro del número inaugural, ya que es susceptible de ser interpretado como un texto programático que persigue afinar la forma como la empresa editorial se representa. Es decir, la revista, de una u otra manera, está tratando de definir una línea editorial que atrape a ciertos consumidores culturales que, por supuesto, se interesen por los tópicos que la publicación aborda. Por último, el artículo actúa como un resumen (salvando las distancias en cuanto a volumen) del trabajo que Richard había elaborado hasta el momento de la creación del proyecto: una ordenación/construcción del mapa cultural "alternativo" postgolpe militar ${ }^{11}$.

Nelly Richard, en ese artículo, realiza una descripción panorámica de la producción cultural que surgió en la dictadura; específicamente, aquella que se valió del "idioma de la transversalidad para contestar" el discurso dominante que pretendía normar, unificar y totalizar las creaciones, las interpretaciones (6). Por ello, Richard se detiene en las producciones culturales — sobre todo, en el ámbito del "arte y la literatura" — que utilizaron una estética experimental con el fin de crear formas expresivas que mantuvieran viva la capacidad problematizante del lenguaje; es decir, estudiar "todas aquellas producciones destinadas a fracturar el mensaje oficial de una verdad única” (6-7). Por lo tanto, la directora menciona a los artistas y a los escritores que se dedicaron a originar discursos culturales desde la insurreccionalidad, desde lo múltiple, desde lo fragmentario, desde lo parcial, desde lo heterogéneo y desde lo inestable, para rebatir la idea de una monoenunciación; tan arraigada, según Nelly Richard, en el contexto político chileno (7). Además, N. Richard aborda explícitamente el problema de los límites (problema que se ve con insistencia en ese número y durante toda la trayectoria de la revista) y, al mismo tiempo, aboga por las creaciones que apuestan por las borraduras de las fronteras disciplinares para recrearlas desde un nuevo lenguaje. De hecho, afirma Nelly Richard que el lenguaje unívoco que promovía la dictadura estaba relacionado con la idea de establecer límites bien definidos, bien demarcados. De igual forma Richard postula que esa "fijeza" a la que estaban sometidos algunos productores culturales — por

interpreta como un nuevo trabajo de recuperación que responde a la intención de no dejar pasar lo que ocurre en eventos o en publicaciones que dialogan con la línea editorial.

${ }^{11}$ Entre sus libros publicados, antes de ese artículo, se encuentran: Márgenes e instituciones (1986) y La estratificación de los márgenes (1989). Estos libros abordan un tipo específico de prácticas culturales que, para decirlo con el título de su escrito, utilizan "estéticas de la oblicuidad”. 


\section{Producir una empresa editorial. El caso de la Revista Crítica Cultural}

ejemplo, los protestatarios-denunciantes con su intención de hacer oposición abierta al régimen militar- impedía una “confusión de signos” y, por ende, imposibilitaba nuevos giros en el lenguaje.

Para N. Richard, el orden del discurso dictatorial reforzaba los límites que separaban y aislaban (discursos, identidades, territorios); límites que prohibían "el tránsito y la errancia” (7). Tránsito y errancia que, tanto a Richard como al proyecto de crítica cultural, le interesa rescatar y que se ve de manifiesto en esas "prácticas insumisas" que "eligieron multiplicar los puntos de intersección y las líneas de fuga entre códigos heterogéneos" (énfasis de la autora). Es en el marco de este análisis donde Richard inscribe a una serie de personajes del ámbito cultural chileno —en la plástica “Altamirano, Díaz, Dittborn, Lotty Rosenfeld”; en narrativa "Diamela Eltit”; en las instalaciones "Leppe, Brugnoli, Errázuriz"; en poesía "Martínez, Zurita, Muñoz y Maqueira" - que ejemplifican la factibilidad, como subraya Richard, de un lenguaje otro que permite cuestionar, interpretar y desarmar discursos. No obstante, lo significativo de este listado son las figuras que participan de la empresa editorial (Eugenio Dittborn, Diamela Eltit y Carlos Altamirano) porque Nelly Richard los reconoce como quienes contribuyeron a minar el lenguaje institucional durante la época militar y, ahora, con el trabajo de editores, contribuyen a articular un nuevo discurso crítico que toma en cuenta las condiciones políticas/sociales/culturales de cambio: la producción en transición democrática. Así como Richard nombra a Dittborn, Eltit y Altamirano también lo hace con las "acciones de arte" de Lotty Rosenfeld, artista que con una videoinstalación inaugura gráficamente la publicación ${ }^{12}$. Estos artistas nombrados por la editora son reconocidos por un trabajo previo pero, por sobre todo, reconocidos por ella, quien les da su aprobación.

Es evidente que con el acto de nombrar a los colaboradores se confirma la intención de resaltar la trayectoria de quienes dan solidez a la publicación. En otras palabras, Richard, en su primer artículo, reivindica producciones y productores específicos. Es decir, se posiciona como vocera de un espectro cultural bien delimitado: aquel que pertenece y sigue el trayecto productivo de la "escena de avanzada". Esto es, se coloca del lado del discurso que vincula arte, cultura y política, pero, desde una perspectiva que no cae en la producción contestataria denunciante sino en aquella que se vale de los “descalabros de la representación” (2006). Richard, con este texto legitima a los productores culturales que la acompañan en la aventura del proyecto editorial. En este punto es necesario aclarar que Nelly Richard no sólo se

\footnotetext{
12 En el primer número de la Revista de Crítica Cultural aparecen, a lo largo de todo el ejemplar, fotografías de una obra-video, expuesta en Berlín (1989), de la artista chilena Lotty Rosenfeld.
} 


\section{Carolina Ramírez Álvarez}

limita a las formas de producción cultural de esa escena sino, también, a otras estéticas experimentales/rupturales que provengan de cualquier espacio geográfico y campo de saber.

Algo similar ocurre en la edición $\mathrm{N}^{\circ}$ 9, donde aparece publicado un artículo de N. Richard titulado "La ropa usada y su estética de segunda mano”, donde hace una reflexión en torno a este tópico y sus posibles significados. Además de la interesante lectura que hace Richard del tema de la ropa usada, en un fragmento del texto llamado "Residuos americanos" se dedica a comentar teóricamente una instalación realizada, en Washington, en 1983, por el grupo CADA. Es decir, Richard respalda su reflexión —de más de tres páginas - con el ejemplo concreto de una obra ejecutada por algunos de los integrantes de la revista, ocurrida mucho antes de su participación como editores. De hecho, la edición $\mathrm{N}^{\circ} 9$ y desarrolla esta línea de razonamiento dedicando las siguientes páginas a dar cobertura a "El caso Simón Bolívar", por lo que se recogen recortes de varios medios de comunicación, en especial artículos de prensa, que se ocupan de la transgresora pintura de Juan Dávila que se dio a la tarea de desmitificar la tradicional imagen del prócer latinoamericano. Dávila pintó a un Simón Bolívar transexual; sin "la compostura marcial, la virilidad militar" y con "pechos de mujer, aros, medias y rouge” (Díaz, Dittborn, Duclos, 29). La revista concede once páginas a la polémica surgida por la obra de uno de los integrantes del equipo editorial. Es decir, nuevamente respalda y genera un discurso crítico sobre las producciones de sus editores; producciones que, obviamente, buscan construir un lenguaje cuestionador de las formas convencionales de representación.

Como los casos descritos anteriormente existen muchos otros a lo largo de la trayectoria de la Revista de Crítica Cultural, debido a que la publicación está colmada de intervenciones que acreditan ciertas producciones. Resultan verdaderamente significativas aquellas que hacen los editores de la revista ya que, gracias a ellas, se puede establecer en forma más clara el orden del discurso que privilegia la empresa editorial. Asimismo, es importante destacar que la legitimación de los modos específicos de hacer cultura viene luego de una reflexión teórica que sustenta Richard, pues de ningún modo los textos que se editan se presentan como meros elogios que pretendan avalar el proceder de los creadores.

En suma, Richard y su proyecto editorial, en el período de transición democrática, refuerzan un modo particular de hacer cultura que surgió en tiempos dictatoriales y que tuvo visibilidad, según ella, gracias a la "escena de la avanzada”. Para la directora de la revista, esta escena se valió de un lenguaje transversal y oblicuo con el objeto de realizar un trabajo de desmontaje de los discursos por los cuales estaba marcado el campo cultural 


\section{Producir una empresa editorial. El caso de la Revista Crítica Cultural}

en la dictadura: aquellos que fomentaban posturas estético-políticas demarcadas y enfrentadas. En otras palabras, esta escena de "explosión cultural" se ubica como una tercera manera que busca producir cultura por medio de un lenguaje transgresor, performático y transdisciplinario. Del mismo modo, la producción de la "escena" se distanció de los espacios formales de discusión (academias, museos, universidades, etc.) para elaborar una propuesta con la intención de corroborar que existen lugares no canónicos donde la productividad es posible; espacios que incluso son valorados por encima de los oficiales e institucionales. Igualmente, es viable afirmar, gracias al reconocimiento que tiene la Revista de Crítica Cultural en la actualidad, que estos personajes que conformaron la "escena de avanzada" y que fundaron la revista, hoy recogen, simbólicamente, las ganancias de una inversión que fue realizada desde la dictadura. Inversión que, por supuesto, el quehacer crítico de Richard trata de rentabilizar al máximo debido a que, como se mencionó anteriormente, ella es quien rescata y le da forma a ese discurso.

\section{ANTECEDENTES EDITORIALES DE LA REVISTA}

Si bien la directora de la publicación establece como antecedente de la Revista de Crítica Cultural a un movimiento político-cultural bautizado por ella como "escena de avanzada", también es cierto que existieron varias aventuras editoriales que pueden ser consideradas un precedente para este proyecto de crítica. Específicamente, en la época dictatorial surgieron diversos intentos de abrir espacios editoriales que le dieran cabida a las producciones críticas y artísticas que ocurrían en el campo cultural "alternativo" chileno. Entre estas publicaciones se encontraba Manuscritos (1975), dirigida por Cristián Huneeus y editada por Ronald Kay, concebida como

la referencia más temprana para las publicaciones y la producción crítica de artes visuales que participan en la rearticulación del campo del arte después del golpe militar. Si bien corresponde a una revista universitaria de literatura y filosofía, la formulación de sus contenidos bajo el concepto de visualización, instaura un modelo editorial que tiene efectos en una serie de publicaciones generadas en la escena plástica a partir de 1976 (Honorato y Muñoz, en línea).

Como se afirma en esta cita, Manuscritos constituye el primer proyecto editorial que se ocupa de las artes visuales luego del golpe del 73. Y, al mismo tiempo, establece una pauta que será seguida por la mayoría de las publicaciones que tienen la cultura/el arte como centro de su apuesta: como es 


\section{Carolina Ramírez Álvarez}

el discurso gráfico, discurso que -como se verá luego- la Revista de Crítica Cultural cuidará en forma detallada. Por otra parte, Honorato y Muñoz arrojan una información valiosa para comprender la transcendencia de las publicaciones periódicas en un campo que "hasta ese entonces (...) no había manifestado la necesidad de documentar su producción de manera consistente" porque aseguran que, de forma eventual, "circulaban folletos subordinados a los eventos expositivos y que constitutivamente no contemplaban el archivo como destino”. En otras palabras, Manuscritos era una revista que se planteaba con una periodicidad regular, única en su estilo y, al mismo tiempo, que venía a contribuir con el registro cultural de un período caracterizado por la ausencia de proyectos editoriales estables. Luego del primer número, esta revista es censurada y se prohíbe su distribución por ser considerada "un escándalo y un atentado al orden", por el rector de la Universidad de Chile de esos años. Manuscritos, como proyecto dependiente de la institución universitaria, se ve obligada a interrumpir, abruptamente, su circulación a pesar de tener el segundo número listo para ser publicado.

Por otra parte, V.I.S.U.A.L. es un sello editorial armado por Eugenio Dittborn que publica Dos textos de Nelly Richard y Ronald Kay sobre 9 dibujos de Dittborn (1976) y Final de pista (1977). El trabajo de V.I.S.U.A.L. se propone como

la primera publicación chilena que da lugar a una nueva escritura de arte, formulada por Nelly Richard y Ronald Kay. Con este acontecimiento editorial se instaura una práctica de trabajo crítico entre artista y teórico, que asume el espacio de la página como registro de creación. En la publicación, la obra de Dittborn es pensada y comentada en el soporte de producción editorial (Honorato y Muñoz, en línea).

Esta cita resulta importante porque evidencia una línea genealógica directa de la Revista de Crítica Cultural. Es decir, Nelly Richard participa, catorce años antes de la fundación de la Revista de Crítica Cultural, en una publicación con Dittborn, integrante del consejo editorial del proyecto. Además, y como elemento fundamental, V.I.S.U.A.L. establece un diálogo importante entre la escritura crítica y el arte, característica que será una constante en la Revista de Crítica Cultural. Entonces, este proyecto funda un patrón editorial que ha de ser seguido por otras publicaciones, entre ellas, Revista de Crítica Cultural.

Luego de la experiencia con V.I.S.U.A.L., Richard trabaja en diferentes empresas editoriales que, por supuesto, serán escuela para la de 1990; entre éstas, Cal (1979), el suplemento La Separata (1982), editado con Fernando Balcells, Eugenio Dittborn y Adriana Valdés y, finalmente, Cuadernos 


\section{Producir una empresa editorial. El caso de la Revista Crítica Cultural}

de/para el análisis (1984), editada con Justo Pastor Mellado (Hernández, 11). Por su actividad constante en el plano editorial, Pablo Oyarzún describe a Richard como una persona "Sostenida e insistente en la publicación de opciones creativas en las artes visuales y en la promoción fidedigna de un modo de escritura que quiere la aprehensión rigurosa de aquéllas” (1987:43).

Resulta evidente, a partir de estos datos, que la trayectoria editorial de Richard no se inicia con la Revista de Crítica Cultural. Importantes ensayos previos dan cuenta de una preocupación de largo aliento que compartió, además, con algunos de los artistas e intelectuales que la acompañarían luego a formar la publicación que aquí se analiza (Eugenio Dittborn y Adriana Valdés). ¿Cuáles serían las razones para la no continuidad de estos proyectos editoriales? No hay certeza de ello, pero Adriana Valdés arroja una explicación que puede haber influido en el breve destino de éstos: "las dificultades de publicación y distribución” (Valdés, 81). En este sentido, Jorge Montealegre, editor de La Castaña, relata que su revista "se distribuía por mano" y como una gran hazaña se llegó a vender en "algunas librerías" $(16)^{13}$. Estos breves testimonios dan cuenta de lo "rudimentario" que podían resultar los procesos de producción y distribución de una revista en la época dictatorial; proyectos editoriales que, obviamente, no contaron con un financiamiento por parte del Estado y que, además, fueron marginados.

Un documento importante para esta investigación, y que ayuda a construir el mapa de las revistas culturales en Chile, a partir de la segunda mitad del siglo XX, es la edición No. 31 de la Revista de Crítica Cultural. Este número está dedicado a recordar, como lo anuncia su portada, varias "revistas literarias, académicas y culturales” que surgieron antes y en los tiempos de la Unidad Popular, la dictadura militar y, finalmente, en el período de la transición democrática. En ese número monográfico se realiza una visión panorámica de otras publicaciones latinoamericanas que, de alguna forma, han trazado un diálogo crítico con la Revista de Crítica Cultural. Araucaria (editada en Francia por chilenos exiliados); Debate feminista (México); Punto de Vista, Confines, H.I.J.O.S. y El Ojo Mocho (Argentina) figuran entre las revistas que son dignas de ser estudiadas, según la revisión que se propone desde las páginas de Revista de Crítica Cultural. Sin embargo, para el desarrollo de este trabajo, interesa detenerse en los proyectos editoriales que hicieron historia en la época dictatorial chilena y que pudieran sentar algún precedente para la Revista de Crítica Cultural, haciendo hincapié editorial.

\footnotetext{
${ }^{13}$ La Castaña fue una “revista de tamaño bolsillo, sobre poesía, gráfica y humor” que circuló “entre los años 1982 a 1987, con 7 u 8 números”, en Chile. Fuente: www.ergocomics.cl (consultado el 8 de septiembre de 2007).
} 


\section{Carolina Ramírez Álvarez}

En la edición de Revista de Crítica Cultural, que se viene comentando, aparecen las dos editoriales de Número Quebrado, revista que se publicó en dos ocasiones, una en septiembre-diciembre de 1988 y otra en diciembre de 1989. Número Quebrado, en su primera edición, definía entre sus objetivos

contribuir a disipar algunas de las muchas carencias que aquejan a la vida y comunicación cultural en Chile. Casi no hace falta referirse a las pésimas condiciones de circulación de los libros chilenos y extranjeros, a la escasez de lectores y lecturas, a la exigua y fantasmática existencia de una crítica literaria, artística, filosófica, musical, etc. que, por lo general, resulta incapaz de dar cuenta de las obras que se producen, rebajada, como se encuentra, a la dimensión de la crónica ínfima, esporádica, insustancial. Tampoco parece necesario consignar nuestro aislamiento cultural; el enclaustramiento de muchos creadores e intelectuales, con la consiguiente autorreferencialidad y provincianismo dogmático y egolátrico; la desinformación respecto de obras y experiencias que ocurren en otros países de América Latina, por no decir nada del resto del mundo; el divorcio entre los (escasos) productores y el (escaso) público; el predominio de publicaciones oficiales y contraoficiales que, si lo hacen, asignan a lo cultural un espacio regimentado, subsidiario y decorativo; la falta de polémica y debate, unida a una cierta abundancia de pequeñas riñas y mezquinas peleas, chismorreos de aldea megalómana; una, a veces, galopante impunidad intelectual (1988:28).

Este panorama de la "comunicación cultural en Chile" descrito por el editor Miguel Vicuña, resulta revelador para entender el precario alcance de las discusiones críticas que se daban en los distintos campos del saber y, al mismo tiempo, permite construir una cartografía de la realidad cultural/editorial previa al surgimiento de la Revista de Crítica Cultural. Según Vicuña, "la cultura” en la época dictatorial estaba sumergida en una especie de "apagón” que no permitía la circulación de saberes, ya sea por las malas condiciones de distribución de los libros, así como por la pobre cantidad de "lectores y lecturas". Igualmente, pone de manifiesto el desconocimiento reinante en el campo cultural chileno con respecto a lo que acontece en otros espacios geográficos. Es decir, reflexiona sobre la necesidad de una puesta al día y de colocar en un lugar privilegiado "a lo cultural” . Por eso, Vicuña con su esfuerzo editorial pretende lograr

la fractura de un espacio posible para la crítica y la escritura, para la polémica y la lectura; la infracción del aislamiento y el provincianismo autoritario; la apertura hacia un intercambio vivo con obras $y$ 


\section{Producir una empresa editorial. El caso de la Revista Crítica Cultural}

expresiones que hoy por hoy se producen en América, Europa y otros países del mundo.

A pesar de las intenciones de irrumpir en el campo cultural chileno de dictadura, Número Quebrado no llega a salir en tiempos democráticos. Es

decir, el intento editorial se consume en la segunda entrega.

Como queda demostrado, el espectro de las publicaciones periódicas en el Chile dictatorial es poco consistente, ya que los intentos de publicación que surgieron se desvanecieron en los primeros números. Tal situación permite afirmar que para el año 90 no existían proyectos editoriales estables que abordaran temas culturales desde una óptica más ruptural, más transdisciplinaria. Es en este contexto que la Revista de Crítica Cultural surge e intenta centrar su tarea en captar a un lector en busca de espacios de reflexión alternativos.

\section{MERCADO CULTURAL EN LA TRANSICIÓN DEMOCRÁTICA}

Se puede afirmar que la Revista de Crítica Cultural, como propuesta de intervención estético-política de un determinado grupo de intelectuales, responde a un momento coyuntural de la historia chilena pero, y más allá de eso, responde a la cristalización de un proyecto alternativo que con las condiciones democráticas puede editarse. Esto quiere decir que se produce una oportunidad cultural que permite capitalizar los diferentes esfuerzos editoriales que se llevaron a acabo en los tiempos dictatoriales. En otras palabras, se comienza a cosechar todo el esfuerzo simbólico y material que se invirtió en el gobierno de Pinochet.

No obstante, si se dieron las condiciones para que naciera la Revista de Crítica de Cultural también era necesario crear un mercado que demandara la publicación ya que, como bien afirma Mabel Moraña, este es un factor importante en la constitución de una publicación porque decreta su “continuidad o desaparición” debido a que compite, como todo objeto, "por el consumidor cultural” (2003:34). En la medida en que el proyecto editorial tuviera lectores/anunciantes garantizados estaría asegurando su existencia.

Para garantizar este lector/anunciante, la revista debía saber a qué “consumidor cultural” estaría apuntando. Del mismo modo, debía construir un perfil editorial que le permitiera la captación de ese público, interesado en leer las opiniones que emanaran de las páginas de la revista. Los ejes temáticos, las citas a ciertos intelectuales, los colaboradores de la publicación, los asesores del consejo editorial podrían interpretarse como el catálogo de contenidos que la empresa editorial está construyendo para atraer a sus receptores. 


\section{Carolina Ramírez Álvarez}

Por ello, en los primeros diez números del proyecto se puede observar cómo fue ese proceso de apelar a un lector específico. Desde sus inicios, la revista fue concebida como "independiente sin patrocinio institucional ni académico”, por lo que debía autofinanciarse. Ese financiamiento debía ser generado por los anuncios publicitarios y por los lectores. Por esta situación, en el $\mathrm{N}^{\circ} 8$, la revista publica un aviso que testifica que "son sus avisadores y suscriptores los que la hacen posible” y, además, exhorta a fortalecer el proyecto "suscribiéndose" (7). Ahora bien, como la mayoría de los proyectos editoriales independientes, la materialización de una revista semestral (impresa a color en papel de alta calidad) y en un formato especial (24 x 33,8 $\mathrm{cm}$ ) debía ser una ardua tarea. De hecho, fue Juan Dávila (artista plástico chileno) quien la "apoyó... en los primeros cuatro números" (Richard, entrevista personal, 2005). Según Richard, la revista pudo consolidarse gracias a esos dos primeros años, porque fue conquistando la confianza de los lectores y anunciantes, confianza que se evidencia al ojear de manera panorámica la publicación.

En el primer número de la Revista de Crítica Cultural sólo dos patrocinadores la apoyan: el Centro de Documentación y Estudios (C.D.E), de Paraguay, y la Editorial de Mujeres Cuarto Propio, de Chile. En el segundo número (noviembre de 1990) la publicación ya cuenta con el soporte de seis anunciantes: el Instituto Francés de Cultura, las publicaciones de FLACSO (Facultad Latinoamericana de Ciencias Sociales), el Fondo de Cultura Económica, La Morada (Centro de análisis y difusión de la condición de la mujer), Punto de Vista (revista argentina dirigida por Beatriz Sarlo) y Espacio' Arte (Complejo Cultural). Ocurre de forma similar en el tercero (abril de 1991), repiten Punto de Vista, Fondo de Cultura Económica, FLACSO y el Instituto de Francés de Cultura junto con la Embajada Argentina y se incorporan: Estudios Públicos, Photofile, Francisco Zegers Editor y Sur Ediciones. De esta forma, la cantidad de anunciantes va en aumento hasta llegar a cuarenta y siete en el número diez. Como ya se mencionó, estos anunciantes ayudan a consolidar financieramente la revista pero, a la vez, regulan su producción. Esto es, la "obligan” a mantener una línea editorial que atraiga al lector que pueda, al mismo tiempo, ser un potencial consumidor de sus productos. Es decir, publicitan en la medida en que el proyecto editorial siga convocando a un determinado lector; en este caso, un lector ubicado en el segmento de la alta cultura, al que le interesen los libros, las revistas, las galerías de arte y los cursos de idiomas, por citar algunos ejemplos.

En suma la Revista de Crítica Cultural es una publicación hecha por intelectuales formados en distintas disciplinas que buscan que sus interlocutores estén a la altura de sus producciones culturales; por tanto, que 


\title{
Producir una empresa editorial. El caso de la Revista Crítica Cultural
}

sean lectores capaces de descifrar y armar las propuestas que emanan de la revista. ${ }^{14}$ El número en aumento de los anunciantes muestra la confianza paulatina que fue adquiriendo la revista para sus aliados comerciales. La puntual periodicidad, es decir, que la revista saliera con la regularidad pautada (cada seis meses) y su alta calidad (desde el punto de vista de lo material y de su contenido) hizo que se consolidara como un proyecto editorial consistente para quienes publicitaban en ella. Pero, además, este proceso de afianzamiento en el mercado de las revistas culturales funcionó para fortalecerla entre sus lectores quienes, obviamente, se dieron cuenta de este crecimiento. Por otro lado, como actores importantes en el sustento de la Revista de Crítica Cultural los articulistas siguieron apostando por un espacio editorial que, si se observa su trayectoria, cada día tuvo más reconocimiento internacional.

\author{
Universidad Simón Bolívar* \\ Av. Anauco, Edif. Montecarlo, Apt-12C \\ Colinas de Bello Monte, Caracas (Venezuela) \\ caroramirez@usb.ve
}

\section{BIBLIOGRAFÍA}

BENJAMIN, Walter. Discursos Interrumpidos I. Madrid: Taurus, 1973.

CABRUJAS, José Ignacio. "Los Ayatolas". Revista de Crítica Cultural 9 (1994): 29.

DÍAZ, Gonzalo; Dittborn, Eugenio y Duclos, Arturo. "Pueblo chico, infierno grande”. Revista de Crítica Cultural No 9 (1994): 31.

ELTIT, Diamela. "La ciudad como campo de batalla cultural". Revisar el pasado, criticar el presente, imaginar el futuro. Nelly Richard (ed.). Santiago: Universidad Arcis, 2004, 100-105.

HERNÁNDEZ, Carmen. Urgencia y emergencia de una nueva postura literaria. Diamela Eltit y Paz Errázuriz. Caracas: Universidad Simón Bolívar (tesis), 1999.

HONORATO, Paula y Muñoz, Luz. "Recomposición de escena 1975-1981. 8 publicaciones de artes visuales en Chile”. http://www.textosdearte.cl/recomposicion/index. consultado el 8 de septiembre de 2007.

\footnotetext{
${ }^{14}$ En realidad, la Revista de Crítica Cultural es una publicación hecha por intelectuales formados en distintas disciplinas que buscan que sus interlocutores estén a la altura de sus producciones culturales, es decir, que sean lectores capaces de descifrar y armar las propuestas que se emanan de la revista. Es necesario acotar que se está trabajando particularmente, en otro artículo, sobre los posibles lectores, posibles consumidores del proyecto editorial.
} 


\section{Carolina Ramírez Álvarez}

MONTEALEGRE, Jorge. “Algunas notas (autocomplacientes y hasta nostálgicas) sobre La Castaña”. Revista de Crítica Cultural No 31 (2005): 16-17.

MORAÑA, Mabel. "Revistas culturales y mediación letrada en América Latina”. Hermes Criollo 5 (2003): 33-39.

MOULIAN, Tomás. Chile actual. Anatomía de un mito. Santiago: LOM, 2002.

NEUSTADT, Robert. CADA DÍA: la creación de un arte social. Santiago: Cuarto Propio, 2001.

OLEA, Raquel. "Políticas de Igualdad, discursos de género". Reflexiones teóricas y comparativas sobre los feminismos en Chile y América Latina. Marcela Ríos (ed.). Santiago: Notas del Conversatorio, 1998. (SD).

OYARZÚN, Pablo. “Crítica; Historia. Sobre el libro Márgenes e Instituciones de Nelly Richard”. Arte en Chile desde 1973. Escena de Avanzada y Sociedad. Nelly Richard (coord.). Santiago: Contribuciones Programa FLACSO, 1987. 43-51.

RAMÍREZ, Carolina. Entrevista a Nelly Richard. Santiago de Chile: Universidad Arcis, 2005

RICHARD, Nelly. “Arte, cultura y política en la Revista de Crítica Cultural”. 2006. Consultado el 12 de agosto de 2007.

http://www.criticacultural.org/presentacion.

RICHARD, Nelly. (ed). Revisar el pasado, criticar el presente, imaginar el futuro. Santiago: ARCIS, 2004.

------- La insubordinación de los signos. Cambio político, transformaciones culturales y poéticas de la crisis. Santiago: Cuarto Propio, 1994.

------- “Ropa usada y estética de segunda mano”. Revista de Crítica Cultural No 9 (1994): 20-24.

------- “Estéticas de la oblicuidad”. Revista de Crítica Cultural 1 (1990): 6-8.

------- La estratificación de los márgenes. Santiago: Francisco Zegers, 1989.

------- "Márgenes e Institución. Arte en Chile desde 1973”. Arte en Chile desde 1973. Escena de Avanzada y Sociedad. Nelly Richard (coord.). Santiago: Contribuciones Programa FLACSO, 1987, 1-13.

------- Márgenes e Instituciones. Arte en Chile desde 1973. Melbourne: Art and Text/ Francisco Zegers Editor, 1986.

RÍOS, Marcela; Godoy, Lorena y Guerrero, Elizabeth. ¿Un nuevo silencio feminista? La transformación de un movimiento social en el Chile posdictadura. Santiago: Cuarto Propio y Centro de Estudios de la Mujer, 2003.

SUBERCASEAUX, Bernardo. “Algunas observaciones sobre la crítica de arte en Chile”. Arte en Chile desde 1973. Escena de Avanzada y 
Producir una empresa editorial. El caso de la Revista Crítica Cultural

Sociedad. Nelly Richard (coord.). Santiago: Contribuciones Programa FLACSO, 1987, 33-38.

SUNKEL, Guillermo. "Campo de investigación y políticas comunicacionales”. Revisar el pasado, criticar el presente, imaginar el futuro. Nelly Richard (ed.). Santiago: Universidad Arcis, 2004, 196-202.

URIBE, Armando. Carta abierta a Patricio Aylwin. Santiago: Planeta, 1998.

VALDÉS, Adriana. "La escritura crítica y su efecto: una reflexión preliminar”. Arte en Chile desde 1973. Escena de Avanzada y Sociedad. Nelly Richard (coord.). Santiago: Contribuciones Programa FLACSO, 1987, 81-90.

VICUÑA, Miguel. “Número Quebrado. Editorial No 1”. Revista de Crítica Cultural No 31 (2005): 28.

ZERÁN, Faride. "Complicidades y silencios de la prensa en dictadura". Revisar el pasado, criticar el presente, imaginar el futuro. Nelly Richard (ed.). Santiago: Universidad Arcis, 2004, 210-214. 